\title{
Impacto da Radiodermatite na estética corporal de pacientes com câncer de cabeça e pescoço
}

Impact of Radiodermatitis on body aesthetics in head and neck cancer patients

Impacto de la Radiodermatitis en la estética corporal de pacientes con cáncer de cabeza y cuello

Bárbara de Sousa Cabral ${ }^{\mathrm{I}}$, Paula Elaine Diniz dos Reis ${ }^{\mathrm{II}}$, Elaine Barros Ferreira ${ }^{\mathrm{III}}$

Resumo: Objetivo: descrever o impacto da radiodermatite na estética corporal em pacientes com câncer de cabeça e pescoço submetidos à radioterapia. Método: estudo exploratório descritivo, do tipo série de casos, realizado no ambulatório de radioterapia de um hospital de ensino. Foram incluídos dez pacientes com câncer de cabeça e pescoço que foram submetidos à radioterapia no período de 2015 a 2017. A coleta dos dados foi realizada com instrumento construído para esse fim e por meio de registro fotográfico. Os dados foram analisados de forma descritiva, apresentando-se o cálculo da frequência. Resultados: todos os pacientes apresentaram sinais característicos de radiodermatite, principalmente os sinais clínicos de epilação (nos homens), hiperpigmentação e descamação seca, que afetam a estética corporal. Conclusão: a estética corporal dos pacientes acompanhados foi alterada em decorrência dos sinais clínicos da radiodermatite. Destaca-se a importância de avaliar essas alterações em pacientes com câncer de cabeça e pescoço submetidos à radioterapia.

Descritores: Neoplasias de Cabeça e Pescoço; Imagem Corporal; Radioterapia; Radiodermatite; Estética

Abstract: Objective: to describe the impact of radiodermatitis on body aesthetics in patients with head and neck cancer undergoing radiotherapy. Method: this is an exploratory descriptive case series study conducted at the radiotherapy outpatient clinic of a teaching hospital. Ten patients with head and neck cancer who underwent radiotherapy from 2015 to 2017 were included. Data collection was performed with an instrument designed for this purpose and through photographic record. Data was analyzed descriptively, presenting the calculation of frequency. Results: all patients presented characteristic signs of radiodermatitis, mainly the clinical signs of epilation (in men), hyperpigmentation and dry scaling, which affect body aesthetics. Conclusion: the body aesthetics of the followed patients was altered as a result of the clinical signs of radiodermatitis. It is important to evaluate these changes in patients with head and neck cancer undergoing radiotherapy.

Descriptors: Head and Neck Neoplasms; Body Image; Radiotherapy; Radiodermatitis; Aesthetics

\footnotetext{
I Acadêmica de Enfermagem, Faculdade de Ciências da Saúde da Universidade de Brasília. Brasília, Distrito Federal, Brasil. E-mail: barbarascabra195@gmail.com, Orcid: 0000-0003-4060-3837

II Professora Associada, Faculdade de Ciências da Saúde da Universidade de Brasília, Brasília, Distrito Federal, Brasil. E-mail: pauladiniz@unb.br, Orcid: 0000-0002-9782-3366

III Professora Adjunta, Faculdade de Ciências da Saúde da Universidade de Brasília, Brasília, Distrito Federal, Brasil. E-mail: elainebf@unb.br, Orcid: 0000-0003-0428-834X
} 
Impacto da Radiodermatite na estética corporal de pacientes com câncer de cabeça e pe... | 2

Resumen: Objetivo: describir impacto de la radiodermatitis en la estética corporal en pacientes con cáncer de cabeza y cuello sometidos a radioterapia. Método: estudio exploratorio descriptivo, del tipo serie de casos, realizado en ambulatorio de radioterapia de un hospital de enseñanza. Incluidos diez pacientes con cáncer de cabeza y cuello que fueron sometidos a radioterapia entre 2015 y 2017. Recolecta de datos fue realizada con instrumento construido para ese fin y mediante registro fotográfico. Los datos fueron analizados de manera descriptiva, presentándose el cálculo de la frecuencia. Resultados: todos los pacientes presentaron síntomas característicos de radiodermatitis, principalmente síntomas clínicos de epilación (en los hombres), hiperpigmentación y descamación seca, que afectan la estética corporal. Conclusión: la estética corporal de los pacientes acompañados fue alterada en decurso de los síntomas clínicos de la radiodermatitis. Se destaca la importancia de evaluar esas alteraciones en pacientes con cáncer de cabeza y cuello sometidos a radioterapia.

Descriptores: Neoplasias de Cabeza y Cuello; Imagen Corporal; Radioterapia; Radiodermatitis; Estética

\section{Introdução}

O câncer de cabeça e pescoço (CCP) engloba um grupo heterogêneo de neoplasias definidas por meio de bases anatômicas do trato aerodigestivo superior, incluindo a cavidade oral, faringe e laringe. ${ }^{1}$ No mundo, foram estimados cerca de 246 mil casos novos de neoplasia de língua e cavidade oral em homens e 108 mil em mulheres, no ano de 2018. ${ }^{2}$ No Brasil, o câncer da cavidade oral figura entre os tipos mais frequentes entre os homens (5\%), sendo esperados cerca de 11.180 casos novos em homens e 4.010 em mulheres, a cada ano do triênio $2020-2022 .^{3}$

Os principais fatores de risco para o desenvolvimento do CCP incluem infecção por papilomavírus humano (HPV) e consumo de tabaco e álcool. ${ }^{4}$ Dentre outros fatores, destacam-se a exposição ao sol sem proteção, associada ao maior risco para o desenvolvimento de câncer de lábio; ${ }^{3}$ condições hormonais, história familiar da doença e ingestão de alimentos iodados, em associação com a neoplasia de tireoide; ${ }^{4}$ excesso de gordura corporal e fatores relacionados à exposição ocupacional. ${ }^{3}$

As principais modalidades terapêuticas adotadas para o CCP são cirurgia, quimioterapia e radioterapia, que podem ser empregadas de forma isolada ou concomitante, a depender do estadiamento do tumor. ${ }^{1}$ A radioterapia é usada para tratar cerca de $80 \%$ dos pacientes com CCP. Apesar do benefício do seu uso e seus enormes avanços tecnológicos, comumente os 
pacientes irão apresentar toxicidades associadas à exposição à radiação ionizante, que afetam negativamente a qualidade de vida. ${ }^{5}$

São toxicidades comuns aos pacientes com CCP submetidos à radioterapia: radiodermatite, mucosite, xerostomia e alteração do paladar. Sua ocorrência e gravidade dependem de vários fatores, tais como: volume e local irradiados, dose total, fracionamento, idade, condições clínicas do paciente e tratamentos associados. ${ }^{6}$ Em razão desses efeitos adversos, o paciente pode lidar com alterações na estética corporal. Alterações na voz, disfagia, dor, tosse intermitente, fadiga, alteração do olfato, ansiedade e depressão são exemplos de graus variados de limitações, bem como perda da autoestima e isolamento social. ${ }^{7}$

Pacientes com CCP geralmente apresentam problemas significativos com a imagem corporal, devido à desfiguração visível e à disfunção orgânica tanto da própria doença quanto do tratamento. ${ }^{8}$ O CCP, dada sua complexidade e localização, envolve características anatômicas e fisiológicas, podendo promover alterações que comprometem a estética corporal, a função relacionada à alimentação, respiração e comunicação, bem como a interação social. ${ }^{9}$

Diversos estudos abordam as mudanças na estética corporal em pacientes com CCP decorrentes da desfiguração que ocorre devido ao tratamento cirúrgico necessário para esses pacientes. ${ }^{8,10-11}$ No entanto, pouco se discute sobre as alterações estéticas em pacientes com CCP submetidos à radioterapia. Considerando que a face e o pescoço são as partes mais visíveis do corpo e que as alterações estéticas resultantes da exposição à radiação ionizante podem afetar a qualidade de vida, é importante conhecer tais alterações nesse grupo de pacientes. Assim, a questão de pesquisa do estudo foi: qual o impacto da radiodermatite nas alterações estéticas apresentadas por pacientes com CCP submetidos à radioterapia?

Dessa forma, o objetivo deste estudo foi descrever o impacto da radiodermatite na estética corporal em pacientes com CCP submetidos à radioterapia. 
Impacto da Radiodermatite na estética corporal de pacientes com câncer de cabeça e pe... $\mid 4$

\section{Método}

Trata-se de estudo exploratório de caráter descritivo, do tipo série de casos, realizado no ambulatório de radioterapia de uma Unidade de Assistência de Alta Complexidade em Oncologia (UNACON) de um hospital universitário do Distrito Federal. Uma série de casos compreende de três a dez casos, que são descritos de forma detalhada, com apresentação das características sociodemográficas e clínicas do paciente, tais como sinais, sintomas e procedimentos terapêuticos utilizados, bem como o desenlace do caso. ${ }^{12}$

Os dados deste estudo decorrem de análise secundária de desfechos apresentados por participantes de um estudo desenvolvido pelo grupo de pesquisa denominado "Intervenções profiláticas e terapêuticas no manejo de radiodermatite em pacientes com câncer”. Eram elegíveis para participar do estudo principal, pacientes que apresentavam diagnóstico médico de neoplasia maligna de cabeça e pescoço com indicação para iniciar radioterapia, com ou sem quimioterapia concomitante. Assim, foi constituída uma amostra de conveniência na qual foram incluídos dez pacientes (casos) com CCP que desenvolveram alterações na estética corporal durante o tempo que estiveram submetidos à radioterapia. Os dados foram coletados no período de 2015 a 2017.

Os participantes foram convidados a participar do estudo pela pesquisadora responsável. A avaliação ocorreu semanalmente, desde a primeira sessão de radioterapia até a conclusão do tratamento, por enfermeiras do ambulatório de radioterapia, responsáveis pelo acompanhamento desses pacientes. A avaliação contemplava o preenchimento do instrumento de coleta de dados, contendo características sociodemográficas (idade, sexo) e clínicas (Classificação Internacional das Doenças [CID], estadiamento, finalidade da radioterapia, técnica da radioterapia, dose total em Gy, dose diária em Gy e número total de frações), por meio de entrevista ou obtenção dos dados do prontuário médico. Adicionalmente, o instrumento 
continha a avaliação da pele do paciente e o registro fotográfico realizado pelas enfermeiras avaliadoras.

As características relacionadas às reações decorrentes da exposição à radiação ionizante foram coletadas durante o tratamento. Para documentar a evolução dos resultados ao longo das sessões de radioterapia, foram fotografadas as principais regiões da cabeça e do pescoço sujeitas à avaliação, semanalmente, durante oito semanas, denominando-se: Semana 1 (S1), Semana 2 (S2), Semana 3 (S3), Semana 4 (S4), Semana 5 (S5), Semana 6 (S6), Semana 7 (S7) e Semana 8 (S8). As seguintes regiões foram fotografadas: região frontal da cabeça e do pescoço, perfil lateral direito, perfil lateral esquerdo e região posterior do pescoço. As fotos foram obtidas de forma padronizada, usando a mesma distância do paciente, mesmo tipo e intensidade da luz e mesma câmera (P510 Nikon) para cada paciente, sendo realizadas no consultório de enfermagem durante consulta semanal. Todos os pacientes foram orientados sobre o registro fotográfico e receberam a informação de que o uso das imagens seria apenas para fins de pesquisa. Adicionalmente, a identidade dos pacientes foi preservada, resguardando a região dos olhos nas imagens fotografadas.

A radioterapia foi realizada utilizando um feixe linac de fótons de $6 \mathrm{MV}$, com planejamento conformacional tridimensional (3D-CRT) e uso de máscara termoplástica em todas as sessões de radioterapia a que os pacientes da amostra foram submetidos. Os dados obtidos foram analisados de forma descritiva, apresentando-se o cálculo da frequência dos casos incluídos.

O estudo foi conduzido de acordo com os padrões éticos exigidos (Resoluções 466/2012, 510/2016, 580/2018, do Ministério da Saúde), tendo sido aprovado pelo Comitê de Ética em Pesquisa, processo CAAE no 24692813.6.0000.0030, número do parecer 610.425, em 09 de abril de 2014. Os participantes que deram a sua permissão por meio do Termo de Consentimento Livre e Esclarecido (TCLE) fizeram parte do estudo. O TCLE continha informações sobre o estudo, potenciais riscos e benefícios, participação voluntária e direitos de saída da pesquisa e 
Impacto da Radiodermatite na estética corporal de pacientes com câncer de cabeça e pe... $\mid 6$

preservação do anonimato, bem como um pedido de autorização para usar documentação fotográfica da área irradiada (Termo de Autorização para Utilização de Imagem).

\section{Resultados}

Foram incluídos dez pacientes com CCP que foram submetidos à radioterapia no período de julho de 2015 a maio de 2017. A média de idade foi de 63 anos. O tipo histológico predominante foi o carcinoma de células escamosas $(n=9,90 \%)$, sendo que apenas um apresentou carcinoma indiferenciado tipo linfoepitelial $(n=1,10 \%)$. Dentre os pacientes acompanhados, nove foram submetidos à quimiorradioterapia $(n=9,90 \%)$; e apenas um, à radioterapia exclusiva $(n=1$, 10\%). Quanto aos fatores de risco para CCP, a maioria referiu ser ex-etilista ( $\mathrm{n}=8,80 \%)$, e dois referiram nunca ter ingerido bebida alcoólica $(n=2,20 \%)$. Um dos pacientes acompanhados relatou nunca ter fumado $(n=1,10 \%)$, dois fumavam $(n=2,20 \%)$, e os demais disseram ser exfumantes $(n=7,70 \%)$. Apenas um dos pacientes acompanhados referiu não ter exposição prévia ao sol $(\mathrm{n}=1,10 \%)$. As características sociodemográficas e clínicas dos casos incluídos estão apresentadas na Tabela 1 .

Tabela 1 - Características sociodemográficas e clínicas dos casos incluídos, no período de 2015 a 2017 - Brasília, DF, Brasil, 2020 (n = 10)

\begin{tabular}{|c|c|c|c|c|c|c|c|c|}
\hline Caso & Idade & Sexo & $\begin{array}{l}\text { CID - } \\
\text { (estadiamento) }\end{array}$ & $\begin{array}{l}\text { Finalidade } \\
\text { RT }\end{array}$ & $\begin{array}{l}\text { Técnica } \\
\text { RT }\end{array}$ & $\begin{array}{l}\text { DT } \\
(\mathrm{Gy})\end{array}$ & $\begin{array}{l}\text { DD } \\
\text { (Gy) }\end{array}$ & $\begin{array}{l}\text { Frações } \\
\text { (dias) }\end{array}$ \\
\hline 1 & 70 & $\mathrm{M}$ & C32 - Laringe (III) & Radical & 3D-CRT & 70,0 & 2,0 & 35 \\
\hline 2 & 76 & M & C01 - Base da Língua (IVa) & Adjuvante & 3D-CRT & 70,2 & 1,8 & 39 \\
\hline 3 & 71 & M & C10 - Orofaringe (IVa) & Radical & 2DRT & 70,0 & 2,0 & 35 \\
\hline 4 & 57 & M & C12 - Seio Piriforme (IVa) & Adjuvante & 3D-CRT & 70,2 & 1,8 & 39 \\
\hline 5 & 52 & M & C04 - Assoalho da Boca (III) & Radical & 3D-CRT & 70,2 & 1,8 & 39 \\
\hline 6 & 77 & M & C10 - Orofaringe (IVa) & Adjuvante & 2DRT & 70,2 & 1,8 & 39 \\
\hline 7 & 54 & M & C10 - Orofaringe (IVa) & Radical & 3D-CRT & 70,0 & 2,0 & 35 \\
\hline 8 & 22 & M & C11 - Nasofaringe (IIIb) & Radical & 3D-CRT & 70,0 & 2,0 & 35 \\
\hline
\end{tabular}




\begin{tabular}{|c|c|c|c|c|c|c|c|}
\hline 9 & 71 & $\mathrm{~F}$ & C05 - Palato (IVa) & Adjuvante & 3D-CRT & 70,0 & 2,0 \\
\hline 0 & 79 & $\mathrm{M}$ & C01 - Base da Língua (IVa) & Radical & 3D-CRT & 70,2 & 1,8 \\
\hline
\end{tabular}

Nota: DD - dose diária; DT - dose total; F - feminino; Gy - gray; M - masculino; RT - radioterapia; 2DRT radioterapia convencional; 3D-CRT - radioterapia conformacional.

Todos os pacientes apresentaram alterações estéticas relacionadas aos sinais da radiodermatite, reação cutânea decorrente da exposição à radiação ionizante. Ao longo das oito semanas de acompanhamento, foi possível observar o desenvolvimento de descamação seca, epilação e hiperpigmentação, características que alteram o aspecto da pele e, por consequência, a estética corporal.

Na Figura 1, são apresentadas as imagens dos Casos 1 a 5, em diferentes momentos no decorrer do tratamento. É possível observar que, ao longo das semanas de tratamento, as alterações cutâneas - e, por sua vez, as alterações na estética desses pacientes — tenderam a piorar de acordo com o acúmulo da dose de radiação recebida.

No Caso 1, vê-se que a hiperpigmentação e o ressecamento da pele do paciente evoluíram com o passar das semanas, conforme pode ser verificado nas imagens 1a (segunda semana de tratamento), 1b (quarta semana de tratamento) e 1c (sétima semana de tratamento). É notória a evolução da reação, sendo mais intensa conforme há aumento de dose acumulada, que, nesse caso, era de aproximadamente 60 Gy na sétima semana (Figura 1c). No Caso 3, o paciente recebeu a mesma quantidade de dose por fração, e é possível verificar as alterações na coloração da pele durante as semanas, tendo apresentado eritema, seguido por hiperpigmentação e ressecamento da pele.

Nos Casos 2 e 4, os pacientes apresentaram hiperpigmentação, ressecamento da pele e epilação, como pode ser visto nas imagens 2c e 4c, quando os pacientes haviam recebido cerca de 45 Gy. No Caso 5, o paciente apresentou as mesmas alterações com dose acumulada inferior, de 36 Gy (5c). 
Impacto da Radiodermatite na estética corporal de pacientes com câncer de cabeça e pe... $\mid 8$
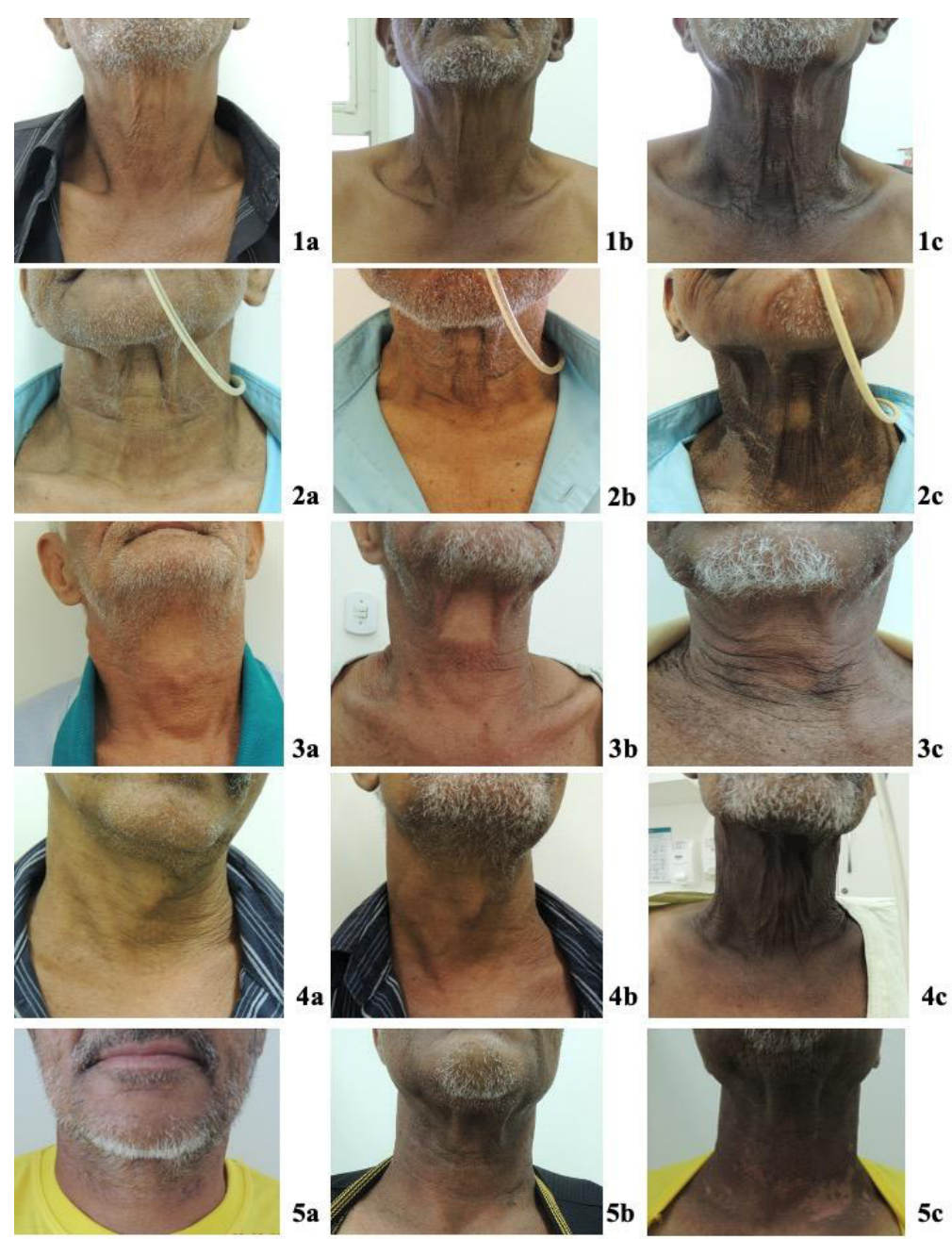

Figura 1 - Acompanhamento fotográfico semanal das alterações estéticas apresentadas pelos Casos 1 a 5 Brasília, DF, Brasil.

Na Figura 2, são mostrados os Casos 6 a 10. A imagem 6a foi realizada no primeiro dia da radioterapia, e a imagem $6 \mathrm{~b}$, na quinta semana de avaliação, quando já havia recebido cerca de 45 Gy de dose cumulativa. Nota-se que o paciente evidencia alteração na coloração da pele, com presença de eritema e hiperpigmentação e, adicionalmente, descamação seca localizada.

No Caso 7, é possível observar que, no decurso das semanas de acompanhamento, o paciente apresentou intensa hiperpigmentação cutânea e descamação seca. A epilação também pode ser observada na face, na imagem $7 b$, realizada na sexta semana de tratamento, quando já havia recebido cerca de 60 Gy de dose cumulativa. 
No Caso 8, a imagem 8 a foi realizada no primeiro dia da radioterapia, e a imagem 8 b, na quinta semana de avaliação, quando o paciente já havia recebido cerca de 50 Gy de dose cumulativa. Vê-se que o paciente apresenta alteração na coloração da pele, com presença de hiperpigmentação, bem como descamação seca e epilação na área irradiada.

No Caso 9, observou-se que, quando a paciente recebeu cerca de 65 Gy de dose acumulada (imagem 9b), mostrou ampla hiperpigmentação da pele, bem como ressecamento da área irradiada.

No Caso 10, verificou-se que, na sétima semana de avaliação (imagem 10b), quando o paciente havia recebido cerca 63 Gy de dose acumulada, ocorreu importante epilação da área irradiada, com hiperpigmentação e descamação seca.

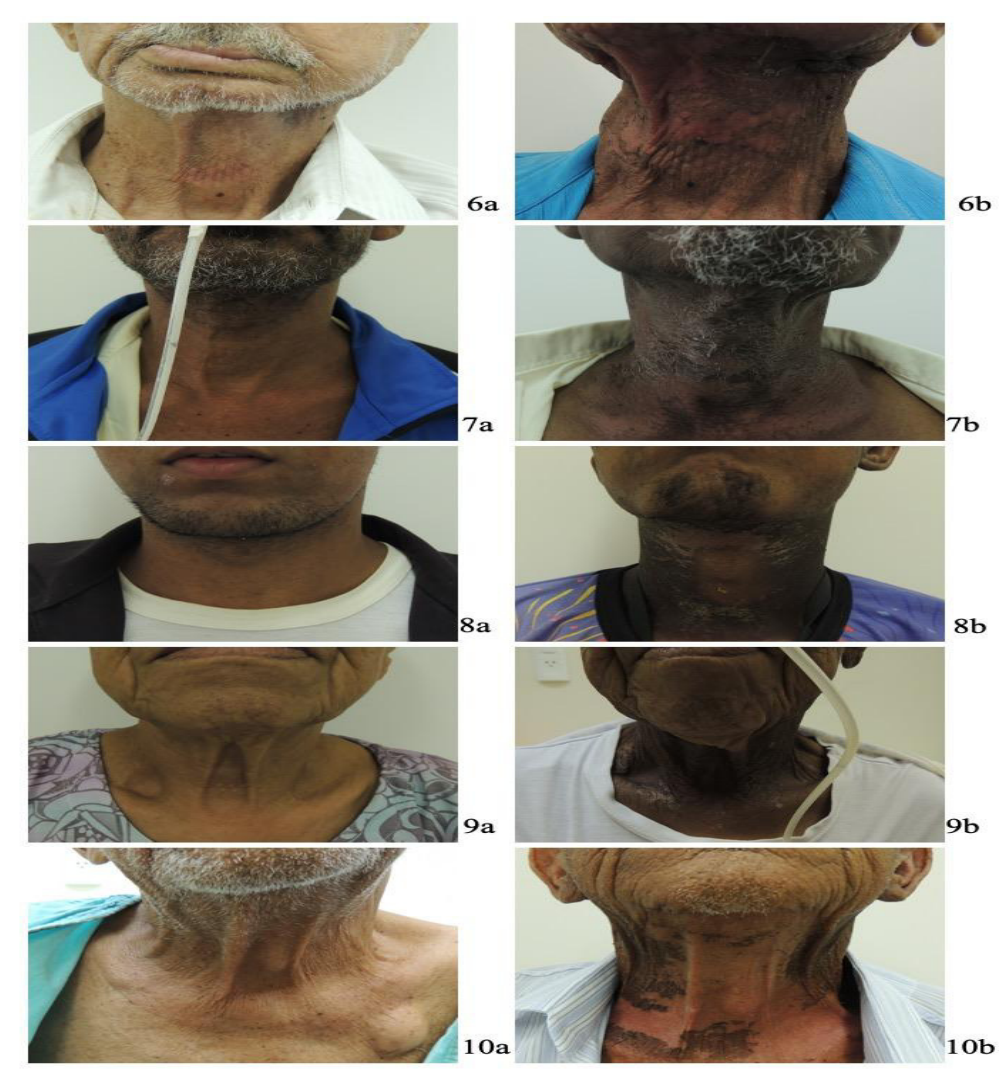

Figura 2 - Acompanhamento fotográfico semanal das alterações estéticas apresentadas pelos Casos 6 a 10 Brasília, DF, Brasil, 2020.

\section{Discussão}

A radioterapia é uma das principais opções de tratamento para pacientes com CCP, com doses variando de 54 a $70 \mathrm{~Gy}$, com fracionamento em torno de 2 Gy/fração e 
Impacto da Radiodermatite na estética corporal de pacientes com câncer de cabeça e pe... | 10

5 frações/semana. ${ }^{13}$ Os efeitos adversos decorrentes da exposição à radiação ionizante podem ter início a partir da primeira dose do tratamento. A cada fração de radiação, maior é a dose acumulada nos tecidos circunjacentes ao tumor e na pele, de modo que esse acúmulo desencadeia uma série de reações inflamatórias decorrentes do maior recrutamento de células inflamatórias para os tecidos afetados com o intuito de diminuir os danos celulares. ${ }^{14}$

A radiodermatite é um tipo de radiotoxicidade desencadeada pelo acúmulo de dose das radiações na pele, órgão que é constantemente afetado por esse tipo de tratamento uma vez que é externo. A graduação da radiodermatite varia em função de fatores de risco relacionados ao tratamento (extrínsecos) e ao paciente (intrínsecos).

Os fatores extrínsecos são: dose total de radiação, esquema de fracionamento da dose, tipo de feixe externo, quimioterapia concomitante, volume e área superficial do tecido irradiado. ${ }^{14}$ Ademais, o uso de dispositivos na área irradiada pode ser considerado um fator de risco, a exemplo da cânula de traqueostomia, que causa umidade e atrito local. ${ }^{15}$

Os fatores intrínsecos são: idade, sexo, tabagismo, doença coexistente, tumor local e fatores genéticos. ${ }^{14} \mathrm{Na}$ série de casos apresentada, verificou-se que houve discreta variação no que concerne à dose total e fracionada da radioterapia bem como ao número de frações. Em relação aos fatores intrínsecos, embora haja mais variações referentes à idade, tipo de tumor e estadiamento, as reações na pele foram muito semelhantes. Apesar da influência dos fatores extrínsecos e intrínsecos, observa-se que a ocorrência das reações na pele são dose-dependentes e seguem um padrão quanto à graduação, iniciando por alterações na coloração da pele, tais como eritema e hiperpigmentação, até a ocorrência de descamação.

O eritema decorre de um processo inflamatório, o qual gera aumento da sensibilidade e pode evoluir com sintomas como edema, secura, queimação, prurido e hiperpigmentação. ${ }^{16} \mathrm{~A}$ hiperpigmentação é um processo pós-inflamatório resultante da ruptura da junção dermoepitelial $^{14}$ que ocorre aproximadamente após duas a três semanas do início da 
radioterapia, com aumento da produção de melanina, ${ }^{16}$ fazendo com que haja um escurecimento da pele. Esse depósito excessivo de melanina nos melanócitos é comum a outras condições cutâneas como sardas, lentigo, cloasma (melasma), descolorações pós-inflamatórias e pós-solares, cicatrizes, reações fototóxicas e fotoalérgicas. ${ }^{17}$ Queixas de alterações de coloração da pele são motivos comuns de consultas dermatológicas em busca de procedimentos estéticos que minimizem esse problema. ${ }^{18}$

A descamação seca é resultante do aumento das taxas de mitose na camada celular de queratinócitos basais, com produção insuficiente de novas células para substituir as da camada basal, desequilibrando o processo de repovoamento das células da pele. ${ }^{14} \mathrm{~A}$ aparência da pele na descamação seca é de opacidade, aspereza e ressecamento, diferente da pele hidratada, que apresenta aspecto suave ao toque, maciez e uniformidade. ${ }^{19}$

Além das alterações estéticas relatadas, destaca-se a perda de folículos capilares, denominada epilação, que ocorre devido a uma diminuição na mitose das células germinativas do folículo piloso. ${ }^{14,20} \mathrm{Em}$ homens, a barba compõe parte da aparência facial, e a mudança gerada pela epilação causa impacto na estética corporal desse grupo de pacientes.

Pacientes com CCP têm alto potencial para distúrbios da imagem corporal, dada a localização altamente visível da doença e os tratamentos direcionados, a exemplo da radioterapia, ainda pouco abordada nesse contexto. A radioterapia pode afetar a aparência física, resultando em edema do tecido (linfedema) e fibrose, que ocasionam mudanças nas características faciais, pigmentação e assimetria facial. ${ }^{21} \mathrm{Na}$ série de casos apresentada, é nítida a alteração da estética facial e da própria autoimagem do paciente, causada pela epilação, alteração da cor e da espessura da pele.

Alterações na estética corporal comprometem o bem-estar psicológico dos pacientes, podendo causar sintomas de ansiedade e depressão, incertezas e baixa autoestima. ${ }^{22-23}$ Como a região da cabeça e pescoço está sempre em exposição, sugere-se que a alteração estética 
Impacto da Radiodermatite na estética corporal de pacientes com câncer de cabeça e pe... | 12

relacionada à ocorrência de radiodermatite afete de forma negativa a qualidade de vida, podendo levar ao isolamento social. Em pacientes com câncer de mama submetidas à radioterapia, avaliadas em um estudo qualitativo, foi observado que a radiodermatite afeta inúmeras dimensões da qualidade de vida e ocasiona distúrbios na imagem corporal. ${ }^{24}$

Pacientes com CCP comumente já enfrentam mudanças físicas significativas no desempenho das funções de fala, respiração e articulação mandibular. Tais mudanças associadas à alteração da estética geram um comprometimento psicossocial. Por essa razão, é fundamental abordar as alterações de imagem corporal ao prestar cuidados a pacientes com CCP desde o início do seu planejamento terapêutico. ${ }^{25-26} \mathrm{~A}$ abordagem de mudanças funcionais e estéticas deve ser sempre considerada pelos profissionais de saúde ao discutirem as intervenções terapêuticas planejadas ao longo da assistência ao paciente, com o intuito de minimizar os prejuízos funcionais e psicossociais por meio de atendimentos prévios com os integrantes da equipe multiprofissional, tais como nutricionista, psicólogo, fisioterapeuta e fonoaudiólogo.

Nessa série de casos, observou-se como os sinais clínicos da radiodermatite podem interferir na estética corporal de pacientes com câncer de cabeça e pescoço. No entanto, comumente se prioriza o enfoque clínico da radiodermatite, avaliando sinais, sintomas e produtos voltados para prevenção e tratamento dessa radiotoxicidade, ${ }^{27}$ sem contemplar o comprometimento estético que pode ocorrer pela reação cutânea. Por sua vez, estudos que abordam alterações na estética corporal de pacientes com CCP observam essencialmente as alterações provocadas por procedimentos cirúrgicos, ${ }^{8,10-11}$ e não propriamente pela exposição à radiação ionizante. Embora pouco estudadas, alterações na estética corporal de pacientes com radiodermatite são reconhecidas como um fator que interfere na qualidade de vida desses pacientes. ${ }^{24}$ Assim, destacou-se, com esta pesquisa, a importância do desenvolvimento de mais estudos que aprofundem o conhecimento sobre esse tema, favorecendo o reconhecimento das 
reações cutâneas como fator que pode interferir na estética corporal de pacientes com câncer de cabeça e pescoço submetidos à radioterapia.

Uma limitação deste estudo foi não avaliar a percepção do paciente sobre a sua imagem corporal. Em estudos futuros, a avaliação da autoimagem pelos pacientes com CCP submetidos à radioterapia pode contribuir para a melhor compreensão do tema. Ademais, destaca-se que o método de série de casos também implica a limitação da não possibilidade de generalização dos dados.

\section{Conclusão}

Nesta série de casos, os pacientes apresentaram sinais clínicos de radiodermatite que afetaram significativamente a estética corporal, a saber: hiperpigmentação, epilação e descamação seca. Considerando o impacto que a alteração da estética corporal pode gerar em pacientes com CCP submetidos à radioterapia pelo fato de haver exposição constante da região, é fundamental que haja planejamento e assistência multiprofissional integrada para oferecer suporte ao paciente ao longo do seu tratamento.

\section{Referências}

1. Yan F, Knochelmann HM, Morgan PF, Kaczmar JM, Neskey DM, Graboyes EM, et al. The evolution of care of cancers of the head and neck region: state of the science in 2020. Cancers (Basel). 2020;12(6):1543. doi: 10.3390/cancers12061543

2. Bray F, Ferlay J, Soerjomataram I, Siegel RL, Torre LA, Jemal A. Global cancer statistics 2018: GLOBOCAN estimates of incidence and mortality worldwide for 36 cancers in 185 countries. CA Cancer J Clin. 2018;68(6):394-424. doi: 10.3322/caac.21492

3. Ministério da Saúde (BR); Instituto Nacional de Câncer José Alencar Gomes da Silva (INCA). Estimativa 2020: incidência de câncer no Brasil [Internet]. Rio de Janeiro: INCA; 2019 [acesso em 2020 out 21]. Disponível em: https://www.inca.gov.br/sites/ufu.sti.inca.local/files/media/document/estimativa2020-incidencia-de-cancer-no-brasil.pdf

4. Silva FA, Roussenq SC, Tavares MGS, Souza CPF, Mozzini CB, Benetti M, et al. Epidemiological profile of patients with head and neck cancer at a cancer center in Southern Brazil. Rev Bras Cancerol. 2020;66(1):e-08455. doi: 10.32635/2176-9745.RBC.2020v66n1.455 
Impacto da Radiodermatite na estética corporal de pacientes com câncer de cabeça e pe... | 14

5. Strojan P, Hutcheson KA, Eisbruch A, Beitler JJ, Langendijk JA, Lee AWM, et al. Treatment of late sequelae after radiotherapy for head and neck cancer. Cancer Treat Rev. 2017;59:79-92. doi: 10.1016/j.ctrv.2017.07.003

6. Lopes RB, Vianna Júnior JJ, França MMC, Sousa EAR, Sousa GA, Mendes EM. Principais complicações orais da radioterapia de cabeça e pescoço: revisão de literatura. Rev Odontol Contemp. 2020;4(1):68-74. doi: 10.31991/v4n12020rocrad

7. Melo Filho MR, Rocha BA, Pires MBO, Fonseca ES, Freitas EM, Martelli Junior H, et al. Qualidade de vida de pacientes com carcinoma em cabeça e pescoço. Braz J Otorhinolaryngol. 2013;79(1):82-8. doi: $10.5935 / 1808-8694.20130014$

8. Hung TM, Lin CR, Chi YC, Lin CY, Chen EYC, Kang CJ, et al. Body image in head and neck cancer patients treated with radiotherapy: the impact of surgical procedures. Health Qual Life Outcomes. 2017;15(1):165. doi: 10.1186/s12955-017-0740-7

9. Formigosa J, Costa L, Vasconcelos E. Representações sociais de pacientes com câncer de cabeça e pescoço frente à alteração da imagem corporal. Rev Pesq Cuid Fundam [Internet]. 2018 [cited 2020 Oct 21];10(1):180-9. Available from: http://www.seer.unirio.br/index.php/cuidadofundamental/article/view/6022

10. Graboyes EM, Hill EG, Marsh CH, Maurer S, Day TA, Sterba KR. Body image disturbance in surgically treated head and neck cancer patients: a prospective cohort pilot study. Otolaryngol Head Neck Surg. 2019;161(1):105-10. doi: 10.1177/0194599819835534

11. Mendes PHC, Barbosa HA, Rodrigues Neto JF, Leite MTS, Sampaio CA. Significado das sequelas faciais estéticas para indivíduos submetidos à cirurgia para tratamento de câncer de cabeça e pescoço. Rev Unimontes Cient [Internet]. 2017 [acesso 2020 out 21];19(1):141-52. Disponível em https://www.periodicos.unimontes.br/index.php/unicientifica/article/view/1589

12. Oliveira MAP, Velarde GC, Sá RAM. Entendendo a pesquisa clínica V: relatos e séries de casos. Femina [Internet]. 2015 [acesso 2020 out 21];43(5):235-8. Disponível em: http://files.bvs.br/upload/S/01007254/2015/v43n5/a5320.pdf

13. Alterio D, Marvaso G, Ferrari A, Volpe S, Orecchia R, Jereczek-Fossa BA. Modern radiotherapy for head and neck cancer. Semin Oncol. 2019;46(3):233-45. doi: 10.1053/j.seminoncol.2019.07.002

14. Singh M, Alavi A, Wong R, Akita S. Radiodermatitis: a review of our current understanding. Am J Clin Dermatol. 2016;17(3):277-92. doi: 10.1007/s40257-016-0186-4

15. Cardozo AS, Simões FV, Santos VO, Portela LF, Silva RC. Radiodermatite severa e fatores de risco associados em pacientes com câncer de cabeça e pescoço. Texto Contexto Enferm. 2020;29:e20180343. doi: 10.1590/1980-265X-TCE-2018-0343

16. Kole AJ, Kole L, Moran MS. Acute radiation dermatitis in breast cancer patients: challenges and solutions. Breast Cancer (Dove Med Press). 2017;9:313-23. doi: 10.2147/BCTT.S109763

17. Lyebyedyeva T, Poticha N, Kiełtyka-Dadasiewicz A. Removal of hyperpigmentation. Evaluation of the efficacy of treatments with tranexamic acid and vitamin C- case report. Arch Physiother Glob Res. 
2019;23(2):21-7. doi: 10.15442/apgr.23.2.3

18. Hollinger JC, Angra K, Halder RM. Are natural ingredients effective in the management of hyperpigmentation? a systematic review. J Clin Aesthet Dermatol [Internet]. 2018 [cited 2020 Oct 21];11(2):28-37. Available from: https://www.ncbi.nlm.nih.gov/pmc/articles/PMC5843359/pdf/jcad_11_2_28.pdf

19. Amaral KFV, Souza RBA. A importância da hidratação cutânea para melhor tratamento das disfunções estéticas. Id On Line. 2019;13(48):763-71. doi: 10.14295/idonline.v13i48.2284

20. Aragüés IH, Pérez AP, Fernández RS. Inflammatory skin conditions associated with radiotherapy. Actas Dermosifiliogr. 2017;108(3):209-20. doi: 10.1016/j.ad.2016.09.011

21. Rhoten BA, Deng J, Dietrich MS, Murphy B, Ridner SH. Body image and depressive symptoms in patients with head and neck cancer: an important relationship. Support Care Cancer. 2014;22(11):3053-60. doi: 10.1007/s00520-014-2312-2

22. Munteanu A, Condorovici D, Patrascu A, Tanase D. Use of gentian violet in the treatment of severe radiodermatitis secondary to adjuvant radiotherapy in breast cancer. Rev Chim. 2017;68(10):2334-6. doi: 10.37358/RC.17.10.5880

23. Rocha DM, Pedrosa AO, Oliveira AC, Bezerra SMG, Benício CDAV, Nogueira LT. Evidências científicas sobre os fatores associados à qualidade de vida de pacientes com radiodermatite. Rev Gaúcha Enferm. 2018;39:e2017-0224. doi: 10.1590/1983-1447.2018.2017-0224

24. Schnur JB, Ouellette SC, Dilorenzo TA, Green S, Montgomery GH. A qualitative analysis of acute skin toxicity among breast cancer radiotherapy patients. Psychooncology. 2011;20(3):260-8. doi: 10.1002/pon.1734

25. Fingeret MC, Teo I, Epner DE. Managing body image difficulties of adult cancer patients: lessons from available research. Cancer. 2014;120(5):633-41. doi: 10.1002/cncr.28469

26. Nayak SG, Pai MS, George LS. Self-image of the patients with head and neck cancer: a mixed method research. Indian J Palliat Care. 2016;22(3):331-4. doi: 10.4103/0973-1075.185050

27. Fuzissaki MA, Paiva CE, Oliveira MA, Canto PPL, Maia YCP. The impact of radiodermatitis on breast cancer patients' quality of life during radiotherapy: a prospective cohort study. J Pain Symptom Manage. 2019;58(1):92-9.e1. doi: 10.1016/j.jpainsymman.2019.03.017

Editora Científica: Tânia Solange Bosi de Souza Magnago

Editora Associada: Rosângela Marion da Silva 
Impacto da Radiodermatite na estética corporal de pacientes com câncer de cabeça e pe... | 16

\section{Autor correspondente}

Elaine Barros Ferreira

E-mail: elainebf@unb.br

Endereço: Laboratório Interdisciplinar de Pesquisa Aplicada à Prática Clínica em Oncologia. Faculdade de Ciências de Saúde Campos Universitário Darcy Ribeiro - Universidade de Brasília - Asa Norte, Brasília - Distrito Federal.

CEP: $70910-900$

\section{Contribuições de Autoria}

\section{1 - Bárbara de Sousa Cabral}

Análise e/ou interpretação dos dados, revisão final com participação crítica e intelectual no manuscrito.

\section{2 - Paula Elaine Diniz dos Reis}

Concepção ou desenho do estudo/pesquisa, análise e/ou interpretação dos dados, revisão final com participação crítica e intelectual no manuscrito.

\section{3 - Elaine Barros Ferreira}

Concepção ou desenho do estudo/pesquisa, análise e/ou interpretação dos dados, revisão final com participação crítica e intelectual no manuscrito.

\section{Como citar este Artigo}

Cabral BSC, Reis PED, Ferreira EB. Impact of Radiodermatitis on body aesthetics in head and neck cancer patients. Rev. Enferm. UFSM. 2021 [Accessed on: Year Month Day]; vol.11 e58: 1-16. DOI: https://doi.org/10.5902/2179769261521 